\title{
MUSE Integral Field Observations of the Oxygen-rich SNR 1E 0102.2-7219
}

\section{Ivo R. Seitenzahl ${ }^{1,2,3}$, Frédéric P. A. Vogt ${ }^{4}$, Jason P. Terry ${ }^{5}$, Michael A. Dopita ${ }^{2}$, Ashley J. Ruiter ${ }^{2,3}$, Parviz Ghavamian ${ }^{6}$ and Tuguldur Sukhbold ${ }^{7}$}

\footnotetext{
${ }^{1}$ School of Physical, Environmental and Mathematical Sciences, University of New South Wales, Australian Defence Force Academy, Canberra, ACT 2600, Australia. email: i.seitenzahl@adfa.edu.au

${ }^{2}$ Research School of Astronomy and Astrophysics, Australian National University, Canberra, Australia.

${ }^{3}$ ARC Centre for All-sky Astrophysics (CAASTRO).

${ }^{4}$ European Southern Observatory, Av. Alonso de Córdova 3107, 7630355 Vitacura, Santiago, Chile.

${ }^{5}$ Department of Physics and Astronomy, University of Georgia, USA.

${ }^{6}$ Department of Physics, Astronomy and Geosciences, Towson University, Towson, MD 21252, USA.

${ }^{7}$ Department of Astronomy and Center for Cosmology \& Astro-Particle Physics, The Ohio State University, Columbus, OH 43210, USA.
}

\begin{abstract}
We have observed the oxygen-rich SNR 1E 0102.2-7219 with the integral field spectrograph WiFeS at Siding Spring Observatory and discovered sulfur-rich ejecta for the first time. Follow-up deep DDT observations with MUSE on the VLT ( $8100 \mathrm{~s}$ on source) reaching down to a noise level of $\sim 5 \times 10^{-20} \mathrm{ergs}^{-1} \mathrm{~cm}^{-2} \AA^{-1}$ spaxel $^{-1}$ have led to the additional discovery of fastmoving hydrogen as well as argon-rich and chlorine-rich material. The detection of fast-moving hydrogen knots challenges the interpretation that the progenitor of $1 \mathrm{E} 0102$ was a compact core of a Wolf-Rayet star that had shed its entire envelope. In addition to the detection of hydrogen and the products of oxygen-burning, this unprecedented sharp $\left(0.2^{\prime \prime}\right.$ spaxel size at $\sim 0.7^{\prime \prime}$ seeing) and deep MUSE view of an oxygen-rich SNR in the Magellanic Clouds reveals further exciting discoveries, including [Fe XIV] $\lambda 5303$ and [Fe XI] 77892 emission, which we associate with the forward shock. We present this exciting data set and discuss some of its implications for the explosion mechanism and nucleosynthesis of the associated supernova.
\end{abstract}

Keywords. supernova remnants

\section{Introduction}

1E 0102.2-7219 (1E0102 for short) belongs to the class of oxygen-rich young supernova remnants (O-rich YSNRs) showing remarkably little-to-no hydrogen emission. The prominent oxygen forbidden-line emission observed in the filaments and knots (see Figs. 1, 2) is understood to arise from shocked ejecta from a core-collapse supernova that had been stripped of (most of) its hydrogen envelope prior to explosion. Unlike most SNRs, where the optical emission comes from interstellar medium ionized by the forward shock, O-rich YSNRs are special in that one can study the actual ejecta composition of the supernova, providing unique and direct insights into explosion mechanisms and nucleosynthesis conditions in core-collapse supernovae. Only a handful of such O-rich 


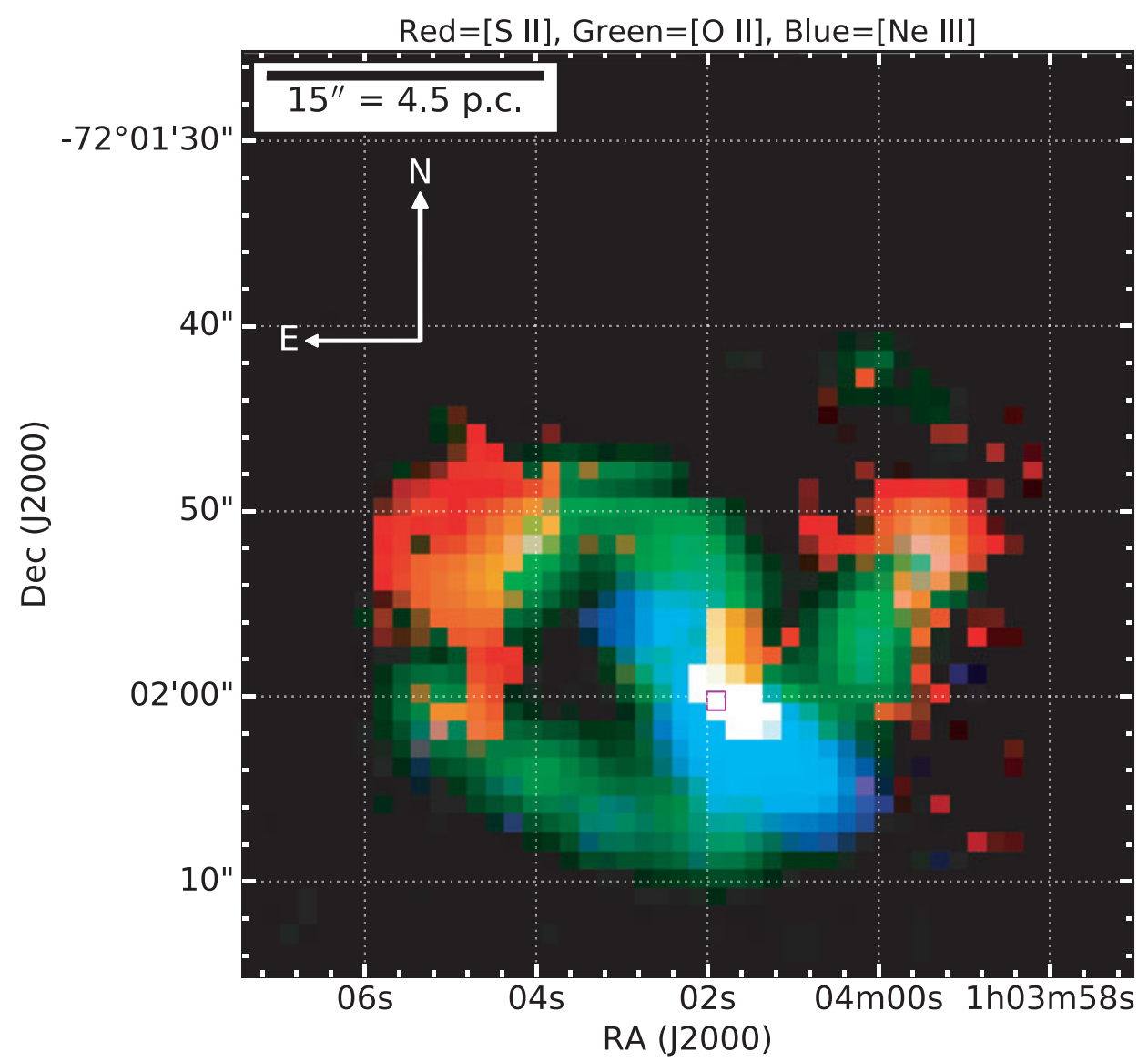

Figure 1. Three color (RGB) image of 1E 0102.2-7219 showing the regions where fast-moving [S II] $\lambda \lambda 6716,6731$ (red), [O II] $\lambda \lambda 3726,3729$ (green), and [Ne III] $\lambda \lambda 38669,3967$ (blue) ejecta is detected with WiFeS.

YSNRs are known: the best studied examples include Cas A in our Galaxy and 1E 0102 in the Small Magellanic Cloud (SMC).

Since the discovery of the high-velocity oxygen-rich supernova ejecta in 1E 0102 (Dopita et al. 1981), subsequent searches (including HST FOS spectroscopy and WFC3 imaging) for the expected products of oxygen burning, in particular sulfur, have returned negative results (e.g., Lasker \& Golimowski 1991, Blair et al. 2000). These searches have only detected carbon, oxygen, neon, and magnesium, but no products of oxygen-burning, i.e., no sulfur, silicon, calcium, or argon. The angular size of $1 \mathrm{E} 0102\left(50^{\prime \prime}\right.$ in diameter $)$ and the large Doppler shifts associated with its fast-moving ejecta $\left(v \gtrsim 1000 \mathrm{~km} \mathrm{~s}^{-1}\right)$ have been strong limitations for previous long-slit spectroscopic and narrow-band imaging searches, which have either failed to map locations of interest and/or were blind to fast-moving ejecta altogether.

\section{WiFeS observations}

In our observations (P.I.: Seitenzahl) on the nights of Aug. 7 and Aug. 8, 2016 with the WiFeS Integral Field Spectrograph on the $2.3 \mathrm{~m}$ telescope at Siding Springs Observatory, we have detected fast-moving (mostly, but not exclusively blue-shifted) [S II] $\lambda \lambda 6716,6731$ 


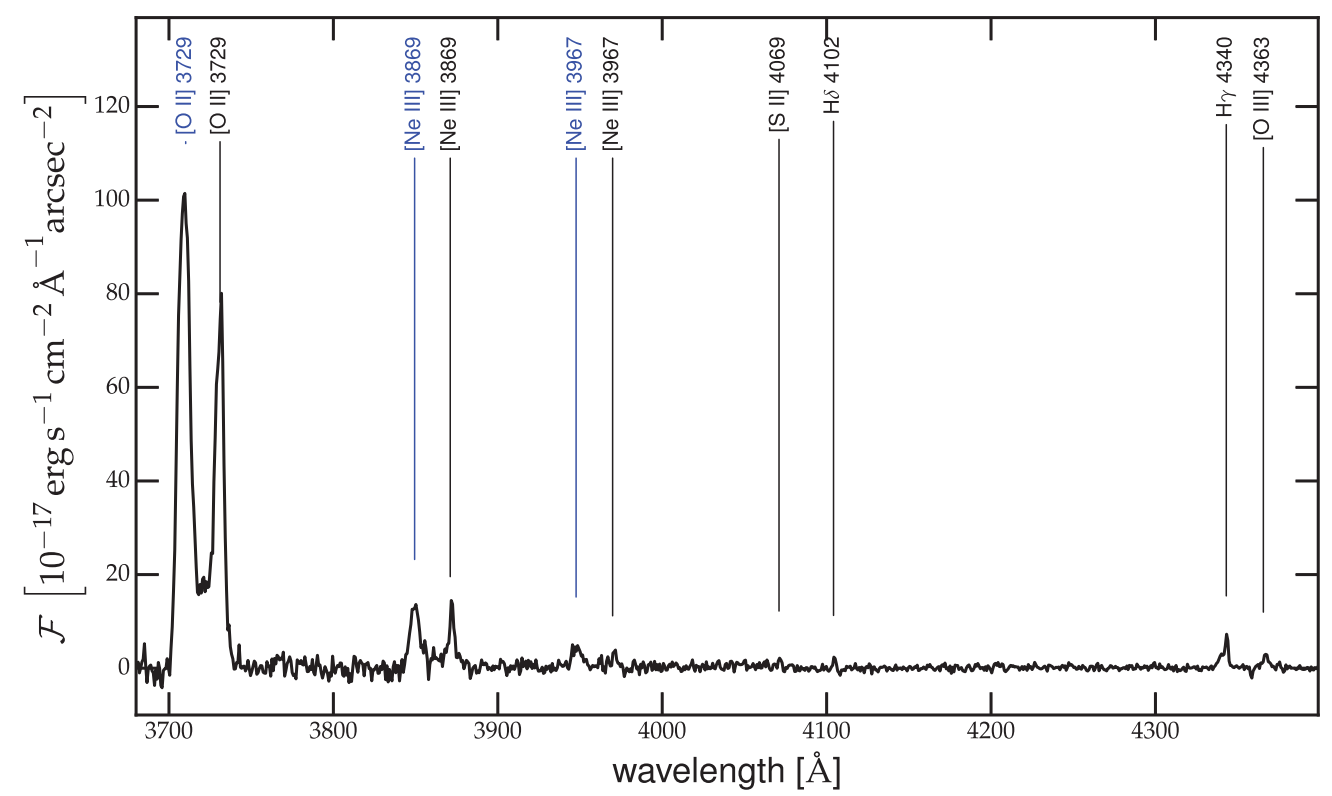

Figure 2. Blue end of the WiFeS spectrum of the bright knot indicated by the purple square in Fig. 1. The broad, fast-moving ejecta component is labeled in blue ([O II $]$ and $[\mathrm{Ne} \mathrm{III]} \mathrm{inaccessible}$ with MUSE), the narrow line emission at the local velocity of $1 \mathrm{E} 0102$ is labeled in black.

emission (see Fig. 1). These data represent the first detection in the optical of the products of oxygen-burning in the ejecta of $1 \mathrm{E} 0102$. The first set of observations were performed with the R7000 and B7000 gratings in place and constituted a co-added $2 \times 1800$ s science observation block for each of the two pointings that make up the mosaic, with a $900 \mathrm{~s}$ dark sky frame before and after. We re-visited the same two fields of the target for an additional $2 \times 1800$ s per science field on the nights of Oct. 28 and Oct. 29, 2016, this time with the B3000 in the blue arm to include [O II] $\lambda \lambda 3726,3729$ and [Ne III] $\lambda \lambda 3869,3967$ (see Fig. 2). All data were reduced and flux-calibrated with the PyWiFeS pipeline (Childress et al. 2014).

\section{MUSE observations}

We observed 1E 0102 with the Multi-Unit Spectroscopic Explorer (MUSE) in Service Mode at the Very Large Telescope on the night of October 7, 2016 via Director's Discretionary Time (DDT) program 297.D-5058 (P.I.: F.P.A. Vogt). We obtained a total of $9 \times 900 \mathrm{~s}$ exposures on-source, with appropriate $180 \mathrm{~s}$ dark sky exposures away from the SMC in between the science exposures. The data frames were individually reduced and then combined into a single data cube with the ESO Reflex workflow (Freudling et al. 2013), for further details see Vogt et al. 2017b. Compared to the $1^{\prime \prime}$ by $1^{\prime \prime}$ spaxel size and $\sim 1.5^{\prime \prime}$ seeing of the WiFeS cube, the $0.2^{\prime \prime}$ by $0.2^{\prime \prime}$ spaxel size and $\sim 0.7^{\prime \prime}$ seeing of the MUSE cube offers a much sharper view of $1 \mathrm{E} 0102$ (see Fig. 3). In addition to gaining in spatial resolution, the MUSE observations have increased the sensitivity by a factor of approximately 100, improving from the WiFeS noise level of a few times $10^{-18} \mathrm{ergs}^{-1} \mathrm{~cm}^{-2} \AA^{-1}$ spaxel $^{-1}$ to a noise level of a few times $10^{-20} \mathrm{ergs}^{-1} \mathrm{~cm}^{-2} \AA^{-1}$ spaxel $^{-1}$. 


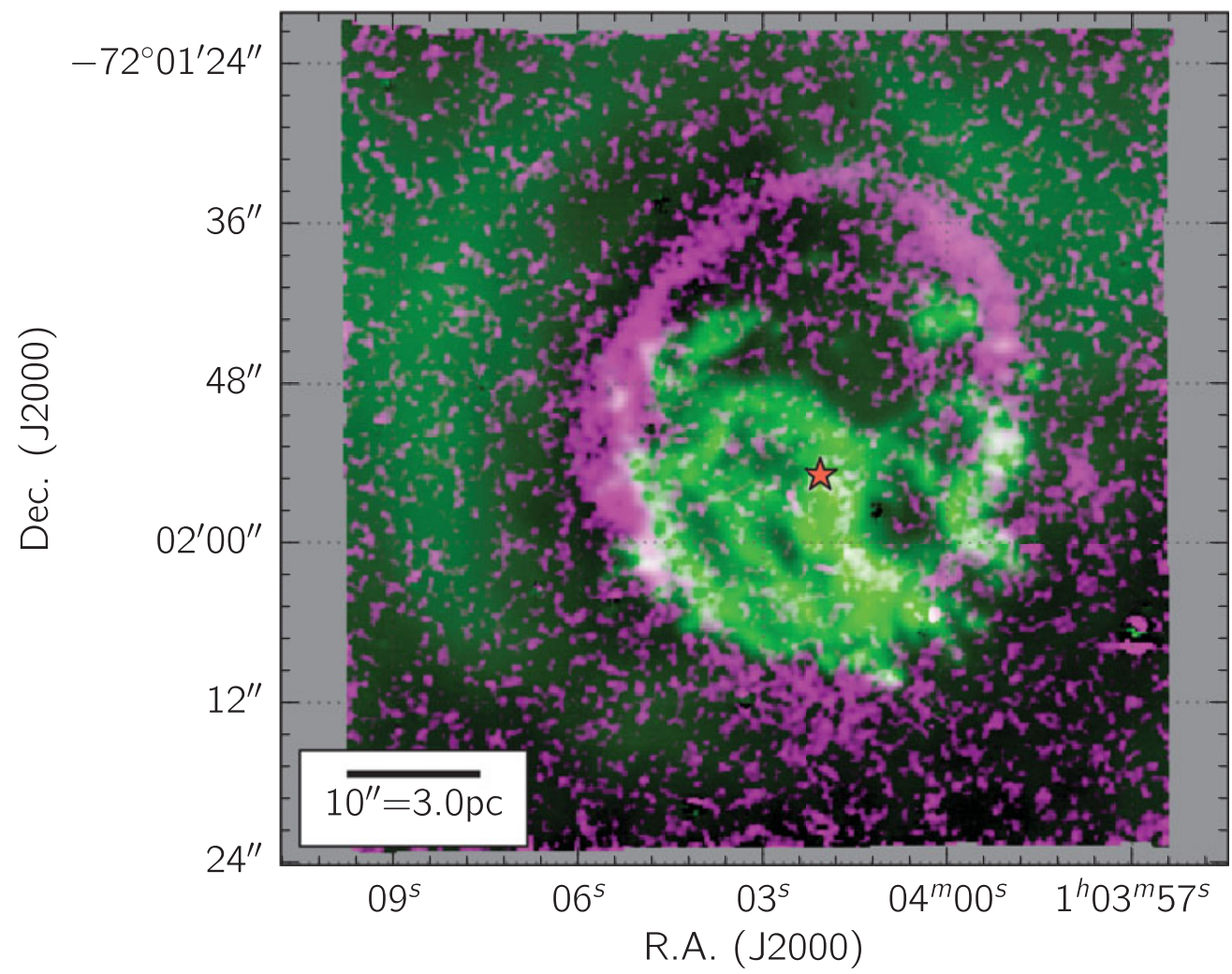

Figure 3. MUSE view of 1E 0102, reproduced from Vogt et al. 2017b. Shown in magenta is [Fe XIV] (5300 - $5308 \AA$ slice through the cube) and in green [O III] (4900 - $5100 \AA$ slice through the cube). The center of expansion derived by Finkelstein et al. (2006) is marked with a star symbol. The clumpy O-rich ejecta is seen in bright green mostly inside the magenta [Fe XIV] ring. The more diffuse [O III] emission outside the magenta [FeXIV] ring is attributed to the photoionization precursor ahead of the forward shock.

\section{Summary}

In this contribution, we have presented optical integral field spectroscopy observations of 1E 0102.2-7219 performed with the WiFeS and MUSE instruments. The combined data cubes extend from $3500 \AA$ to $9350 \AA$. In addition to mapping out the (previously known) O- and Ne-rich ejecta in unprecedented detail, we have discovered fast-moving [S II], [S III], [Ar III], $\mathrm{H} \alpha, \mathrm{H} \beta$, and possibly [ClII], which we attribute to be components of the supernova ejecta. S, Ar, and $\mathrm{Cl}$ are main nucleosynthesis products of O-burning, which we detect here as ejecta in the optical in 1E 0102 for the first time. The discovery of Balmer line emission in the ejecta indicates that the progenitor star must not have been entirely stripped of its hydrogen envelope when it exploded. We have also discovered high ionization coronal lines of $\mathrm{Fe}([\mathrm{Fex}],[\mathrm{FexI}]$, and [Fe XIV]) and the photoionization precursor emission, which can be used to derive the forward shock parameters (see Vogt et al. 2017b).

\section{Acknowledgements}

This research has made use of BRUTUs, a Python module to process data cubes from integral field spectrographs hosted at http://fpavogt.github.io/brutus/. For 

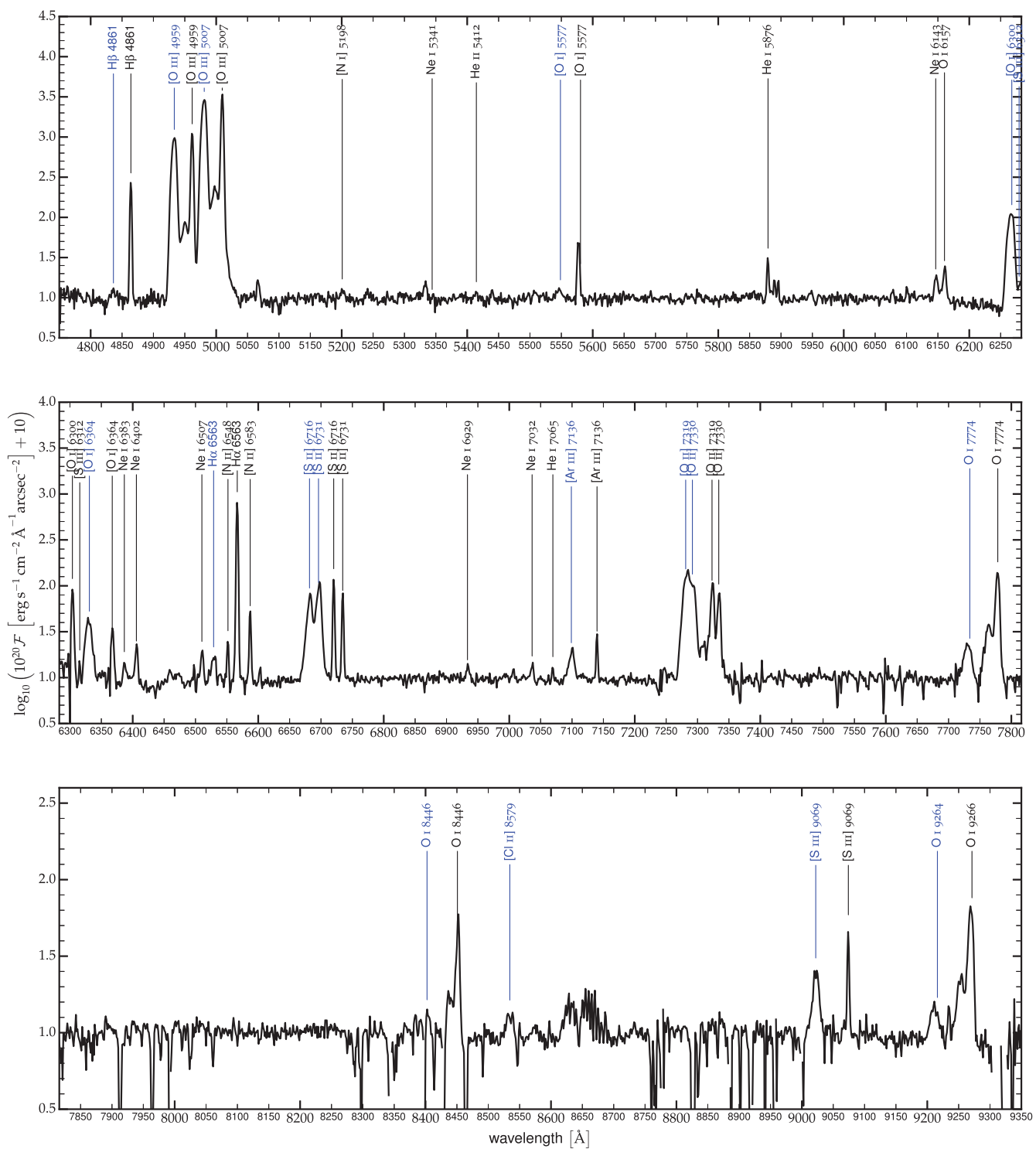

Figure 4. MUSE spectrum of the same S-bright knot as for the WiFeS spectrum in Figs. 1 and 2 . The broad, fast-moving ejecta component is labeled in blue, the narrow line emission at the local velocity of $1 \mathrm{E} 0102$ is labeled in black. Note in particular the clear detections of blue-shifted $[\mathrm{S} \mathrm{II}] \lambda \lambda 6716,6731,[\mathrm{~S} \mathrm{III}] \lambda 9069,[\mathrm{Ar} \mathrm{III}] \lambda 7136$, and the (marginal) detection of $[\mathrm{Cl} \mathrm{II}] \lambda 8579$. Weak lines of $\mathrm{H} \alpha$ and $\mathrm{H} \beta$ are seen at the same Doppler shift.

this analysis, BRUtus relied on STATSMOdel (Seabold \& Perktold 2010), MATPlotliB (Hunter 2007), Astropy, a community-developed core Python package for Astronomy (Astropy Collaboration 2013), APLPY, an open-source plotting package for Python hosted at http://aplpy.github.com, and MONTAGE, funded by the National Science Foundation under Grant Number ACI-1440620 and previously funded by the National Aeronautics and Space Administration's Earth Science Technology Office, Computation Technologies Project, under Cooperative Agreement Number NCC5-626 between NASA and the California Institute of Technology. This research has also made use of the ALADIN 
interactive sky atlas (Bonnarel et al. 2000), of SAOIMAGE DS9 (Joye \& Mandel 2003) developed by Smithsonian Astrophysical Observatory, of NASA's Astrophysics Data System, and of the NASA/IPAC Extragalactic Database (NED; Helou et al. 1991), which is operated by the Jet Propulsion Laboratory, California Institute of Technology, under contract with the National Aeronautics and Space Administration. IRS was supported in part by the Australian Research Council Laureate Grant FL0992131 and the Future Fellowship Grant FT160100028. AJR acknowledges funding through the ARC Centre of Excellence for All-sky Astrophysics (CAASTRO) through project number CE110001020. PG thanks the Stromlo Distinguished Visitor Programme. FPAV and IRS thank the CAASTRO AI travel grant for generous support. Based in part on observations made with ESO Telescopes at the La Silla Paranal Observatory under programme ID 297.D5058[A] and the ANU $2.3 \mathrm{~m}$ telescope at Siding Spring Observatory.

\section{References}

Astropy Collaboration, Robitaille, T. P., Tollerud, E. J., et al. 2013, A\&3 A, 558, A33

Blair, W. P., Morse, J. A., Raymond, J. C., et al. 2000, ApJ, 537, 667

Bonnarel, F., Fernique, P., Bienaymé, O., et al. 2000, A\& A Supplement Series, 143, 33

Childress, M. J., Vogt, F. P. A., Nielsen, J., \& Sharp, R. G. 2014, ApSS, 349, 617

Dopita, M. A., Tuohy, I. R., \& Mathewson, D. S. 1981, ApJ Lett., 248, L105

Finkelstein, S. L., Morse, J. A., Green, et al. 2006, ApJ, 641, 919

Freudling, W., Romaniello, M., Bramich, D. M., et al. 2013, A\& A, 559, A96

Hunter, J. D. 2007, Computing in Science and Engineering, 9, 90

Joye, W. A. \& Mandel, E. 2003, in Astronomical Data Analysis Software and Systems XII ASP Conference Series, Vol. 295, 489

Helou, G., Madore, B. F., Schmitz, M., et al. 1991, Databases and On-line Data in Astronomy, 171,89

Lasker, B. M. \& Golimowski, D. A. 1991, ApJ, 371, 568

Seabold, S. \& Perktold, J. 2010, in Proc. of the 9th Python in Science Conference, 57-61

Vogt, F. \& Dopita, M. A. 2010, ApJ, 721, 597

Vogt, F. P. A., Seitenzahl, I. R., Dopita, M. A., \& Ruiter, A. J. 2017a, PASP, 129, 058012

Vogt, F. P. A., Seitenzahl, I. R., Dopita, M. A., \& Ghavamian, P. 2017b, accepted for publication in $A \&$ A Lett., arXiv:1705.04322

\section{Discussion}

KIRSHNER: It's great you finally found [S II] - in a way we always knew it had to be there. You should try to put a limit on [CaII]. 